\title{
Emergency-cloud - a new idea about the construction of emergency platform in China
}

\author{
Yi liu,Lihua Li,Hui Zhang \\ Institute of Public Safety, Tsinghua University \\ Peking,China \\ E-mail: liuyi789@tsinghua.edu.cn
}

\begin{abstract}
-a new idea about the emergency platform application mode- Emergency-cloud was putting forward based on the status of emergency platform in china. The feasibility of construct it was analyzed and the architecture of the Emergency-cloud was built from bottom to up.at last, the features of the application mode was sort out.
\end{abstract}

Keywords-Emergency-cloud; emergency platform; information systems; cloud computing

\section{INTRODUCTION}

The rapid development of globalization and the wide use of Internet, Facebook and etc. not only short the distance of the social ties and bring the convenience for the world, but also make the social networks to become more complex and fragile. The complexity of the social structure which brought by the globalization greatly increased the difficulty of the government's emergency management. To construct a public safety information platform which could meet the needs of emergency missions today are the current social work of the government in the worldwide. In China, improving the emergency management information level and effectively use the resources brought by the globalization to promote the Emergency Management development are also the important task in the field of public safety.

\section{THE NEW REQUIREMENTS FOR THE EMERGENCY MANAGEMENT PLATFORM}

Emergency disasters have the features of randomness, uncertainty and evolution, and its effective response always needs the coordination of varies of departments and multitypes of resources. In the new information environment, due to the rapid spread of the Internet, relevant state of affairs and information are more easily to be spread and ferment and the various crises are more easily lead to unexpected events. The development of information technology has brought social network coupling with the disaster physical network, so as the dealing with the disaster in the new information environment has become more difficult. Under the new information environment, the complexity of modern social network and the use of multimedia increased the complexity and difficulty of the responds of the emergency management. By the influence of new information technology, no matter the disaster, the disaster carriers and environment, and emergency management, which all have their specific projection in view of the emergency information platform, and all require the emergency response of high efficiency and high adaptability, including highperformance emergency computing services. The application of new information technologies put forward new demand for the construction of the emergency management platform.

Now in china, the governments and departments at all levels and places have established their own emergency responds platform (ERP). (Figure 1) The emergency platform is an integrated emergency business platform for the disaster responding. And in the existing management system of the emergency platform, the power of the government is the dominant. The structure of the platform is based on the power level and division of labor.

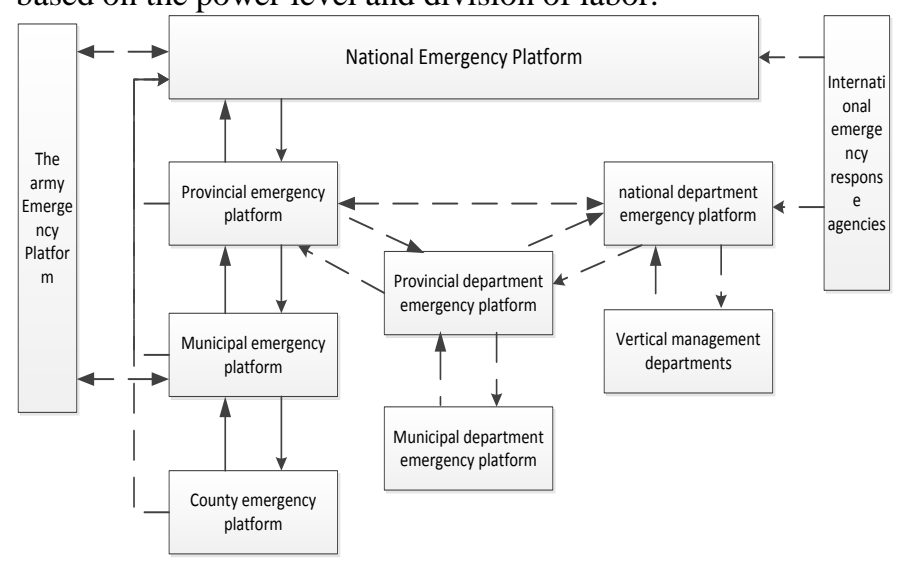

Figure 1 the current ERP in china

The current structure of the ERP is consistent with the administrative level system in china. And when the disaster occurs, it will call the relevant departments of the platform to response. In some sense, the administrative level organizational structure could ensure the implementation of the emergency powers .But meanwhile as the organizational structure now always take the way of only communication interconnect, the information and resources of it are in a state of isolation, dissection static state and localized management. When facing the enormous, complex and dynamic emergency processing task in the new information environment, it is usually difficult to make a correct evaluation on the state of affairs and deploy resources reasonably in time under the intersected pattern the effect of it on resource and information sharing is not very good.

So in the new information society, the increasing complexity of the social network under the new information environment has put forward deep demand for the capability of emergency platform such as:

(1) The multi-source, heterogeneous and complex 
source of information proposed requirements for the capability of computing and storage

Because of the influence of social media and networking under the new information environment, unexpected events occur more suddenly, evolve more rapidly and influence more greatly. The scientific and effective solution to cope with it is to integrate not only the information from the online world, but also the physical world information collected from the Internet of Things to provide real-time, full information for decision-making. The multi-source, heterogeneous and complex source of information from different place, department and time proposed requirements for the capability of computing and storage.

(2) The dynamic feature of the emergency procedure, the real-time characteristic of the emergency decision put forward demand for the capability of service provide

Emergency management has a strong scene dependency, which should be adjusted rationally according to the evolution of the scenario and dynamic changes of resources. Under the new information situation, the occurrence of the disaster is becoming more uncertainty due to the online media participation, so the effective emergency management requires emergency service and applications could be pushed to the front of the decision-makings for the use of fitted departments in the real time. The dynamic features of the emergency management put forward demand for the capability of service provide.

\section{A NEW IDEA ABOUT THE APPLICATION OF CLOUD COMPUTING IN THE EMERGENCY PLATFORM}

\section{A. Feasibility}

A complete and sound emergency management platform is not just an information platform, command platform, but a servant platform for the whole process of emergency management. And the deepen research of the new information technology has put forward new areas for the capabilities of the emergency platform. Facing with the current demand for the construction of the emergency management platform, in the worldwide, Ward \&Wamsley(2000) studied on the evolution of Federal Emergency Management Agency's (FEMA) network. And Harrald(2006)analyzed the reasons why National Incident Management System failed to response the hurricane Katrina, and give an opinion that agile reconstruction and order is the key factors of the emergency responding successfully. In China, Fu \&Liu (2007) and Fu (2008), Rong (2009) think that emergency process should be able to adjust to the emergency management activities. Fan \&Yuan(2006)pointed out that the building of the emergency platform should be closely integrated with the work flow of emergency management. On the new information emergency platform under the information society, Li, Xie \&Liu (2011) pointed out that an emergency system which has the characteristic of providing on-demand and serving dynamically could be built on the cloud-system. And Ren and Wang(2012)analysis of the characteristics of cloud computing, and designed a security and emergency prediction and warning system by the cloud computing architecture. And the researchers gives an opinion that the new information technology such as the cloud computing technology is an effect solution to realize dynamic and integrated emergency management system. And by integrating the dispersed resources idle in the "cloud", the cloud computing has powerful computing capability and cloud resources could be infinitely expandable, and demand to use and enjoy "(Li,Zhang\&Wang,2010) .

So, in this paper, basing on the characteristics of cloud computing, we analysis the feasibility and applicability of cloud computing in the field of emergency management as follows:

(1) High-performance on computing and storage for massive emergency information

(2) The unified management of data for the application of dispersion emergency resources

(3) Provide services according to the emergency task needs

(4) Convenient and green

The characteristics of cloud computing such as demand to use (all services), easy scalability and highperformance of computing, storage and service optimization capabilities are all helped to resolve the information collection, storage, processing and utilization problems in the emergency management.

\section{B. Architecture}

Combine the cloud computing technology and plan a decision-making emergency service system which could provide on-demand services basing on the Integration of resources and meet the requirements of application for the emergency management under the network information environment is a new way for the development of the emergency platform. So build the architecture of emergency platform basing on the cloud computing like firture2.which we could call it Emergency-cloud.

The Emergency-cloud system is an application mode of emergency business which basing on the use of cloud computing technology. Emergency-cloud system is consists of Cloud provider (emergency resources service provider) cloud requestor (the emergency resource service demander) and the Emergency-cloud platform (middleware). Emergency-cloud platform integrate the emergency resource collecting and providing from the monitoring and control platform of Things of internet or internet in real time to construct Emergency-cloud pool. And By unified management of all kinds of physical and network emergency resources, the emergency-platform could provide efficient and reliable emergency service to meet the different emergency requests. And the architecture of Emergencycloud including the physical resources layer information collection layer, emergency-cloud computing platform layer, 
emergency-cloud services layer, emergency response subclouds layer, cloud interface and user layer.All the levels of emergency-cloud listed above could provide technical support from down to the up, and answer the call of the resource from up to down to support for the real-time online emergency decision making.

(1) Physical resources layer and informational collection layer: Through the way of information gathering and physical perception of the internet of things, the real-time monitoring information of emergency scenarios in the physical world and online could be access. The information collection layer stores massive heterogeneous data of events as well as decision-supporting information in the dispersed state; the information collection layer could provide accessible support for the network management of resource along with balanced configuration of Emergency-Cloud. It also provides Preliminary support for the virtualization of the Emergency-cloud resources.

(2) Emergency-Cloud computing platform layer: This layer includes resource pool and the virtualization of cloud resource management and scheduling module. The Emergency-cloud resource pool could realize the collection and merge of big data which is dispersed distributed on the network resources layer logically by the technologies such as virtualization and semantic web. And the Emergency-cloud resource pool also uses the unified classification management to provide support for functional needs of emergency process together. In this layer, the decisionsupport resources such as flexible and high-performance computing, spatial data management and storage, distributed paralleling storage, as well as data, cases and models for the applicable service of upper Emergency-Cloud could be provided by Virtualization and scheduling technology.

(3)Emergency-cloud service layer: This layer transform the emergency-cloud resources into emergency-cloud services by using the precise semantic service packaging technology, and managed and published the service by a unified services registered facilities. Emergency-cloud services layer includes services for emergency application which is transformed from the demand of specific emergency business decision ,such as artificial social modeling, parallel simulation, plan management, scheme generation, comprehensive judgments and etc. and service for emergency management such as SOA component management, service interface management, service registration, the process combination management and etc. and the services for system management such as the cloud user management, resource management, task management , security management and etc.

(4) Emergency response sub-clouds layer: This layer includes static sub-clouds and dynamic sub-clouds. The static sub-cloud was respond to emergency function dimension and emergency application dimensions. Facing the conventional function dimension requires such as monitoring, prediction and warning, simulation, comprehensive judgments, decision-command, operation scheduling and other emergency capabilities to establish the sub-cloud to meet the need of Emergency response function;
Similarly, to establish the sub-cloud to meet the need of Emergency applications needs of emergency response for natural disasters, accidents, disasters, public health emergencies, social security events. The dynamic sub-clouds will establish to response the requirements of the incidents. By dynamically matches the contingency cloud services, which are based on the precise semantics, the dynamic subclouds give requestor supporting by providing timely and efficient cloud services.

(5) Cloud interfaces and user layer: By standardized service access, cloud interface layer will provide service delivery interface to the emergency management platform of all levels of government or various areas of expertise. By terminal access the Emergency-cloud services, different levels of users are able to perform emergency duties.

\section{THE FEATURES LOGIC OF THE EMERGENCY-CLOUD}

The emergency -cloud system is a service supported platform consistent with the rule of request-answer: it support emergency information resources management and agile service support of emergency response on the whole life cycle of emergencies from two dimensions: First, the functions dimensions of emergency process like monitoring and control, early warning forecast, simulation, comprehensive judgments, decision and command, operation scheduling and emergency response; the other, the emergency application dimension like natural disasters, accidents, disasters, public health emergencies, social security. The emergency -cloud system could meet these two dimension emergency respond task accompanied by the life cycle of the emergency -cloud from the occurrence to disappear.

In order to achieve the goal of emergency respond on real-time and online. Emergency-cloud merges technologies like the Cloud Computing, the Internet of things, service oriented, high-performance computing and decision support to manage emergency resources in a uniform and intelligent way and makes them fictionalizing and serviced to meet the demand in the emergency process. This mode not only changed the resource utilization effect of Chinese traditional emergency platform, improve the ability of emergency information system, but also realize universally applicable and efficient in sharing and collaborating, achieving a winwin situation for all the parties in the emergency. The platform has the following characteristics compared with traditional platform in china:

(1) Integrated and intelligent emergency resource management; Emergency-cloud platform integrate the emergency resources dispersed and manages them in a uniform and intelligent way. And by the intelligent emergency-cloud resource pool, the Emergency-cloud platform can provide virtualization management, service package of the data, models, case, and knowledge resources for the demands of all levels, departments, and various types of request. It could provide support for the successful transformation of emergency management applications from concept to reality, and provide a new idea of exploiting 
resources efficiently in the process of emergency management applications.

(2) Dynamic and agile emergency response; Around the demand for the real-time emergency response of the full life cycle of emergency process of from prevention, preparedness, dynamic response to the recovery stage, the emergency-cloud could provide service by the sub-cloud's build on-demand - operate efficiently - reconstruct immediately - disintegrate timely ". This mode has intelligent characteristics of flexible, efficient and. agile. As a serviceoriented, Emergency-Cloud is not simply a computing platform, but a new kind of intelligent emergency management mode in the cloud environment.

\section{CONCLUSION}

Emergency - cloud is a new application mode for the emergency platform in china.it is put forward to solve the problems like the big data, the complex resource scheduling and real-time decision-making in the emergency management process. In this paper, the existing emergency management platform application status and the demand of the new emergency platform in china were analyzed. And the logical view of construction of the emergency-cloud was give and the feature of emergency-cloud was discussed.

\section{ACKNOWLEDGMENT}

To thanks for the project assistance funds: the National Natural Science Foundation. (NNSF:91024032, 91224008; CPSF:2013M540977) in china. And Thanks for the project members working together in Tsinghua University and Jinan University and all the authors cited.

\section{REFERENCES}

[1] Bohu Li, Lin Zhang \& Shilong Wang. (2010) Cloud manufacturing new service-oriented networked manufacturing mode. Integrated Manufacturing Computer Systems, 1,1-7.

[2] Congdong Li,Tian Xie \& Yi Liu. (2011)Cloud-Emergency - the Intelligent Emergency Management new mode. Emergency Management in China,6,52-57.

[3] Gang Ren,Guodong Wang. (20120The design and application of an emergency warning system for the security produce on cloud computing architecture.software.1-4.

[4] Jinchao An,Yueqiang Fu \& Weidong Liu.(2007) Reliability of the emergency system response based on the Workflow. Electromechanical product development and innovation ,1,52-56.

[5] John R \& Harrald (2006) Agillty and Discipline:Critical Success Factors for Disaster Response. The Annals of the American Academy of Political and Social Science ,604,256-272 .

[6] Lei Ren,Lin Zhang\&Yabin Zhang.(2011) Research on the Manufacturing Resource virtualization. Integrated Manufacturing Computer Systems, 3,511-518.

[7] Mei Rong.(2009) Adaptive management in our emergency management. Research of the Development,8,78-81.

[8] Robert Ward, Gary Wamsley, Aaron Schroeder\& David B. Robins. (2000) Network organizational development in the public sector : A case study of the Federal Emergency Management Administration (FEMA) . Journal of the American Society for information Science, 51 (11), 1018-1032.

[9] Weicheng Fan \&Hongyong Yuan.(2006) Status Analysis and Countermeasures of China's construction of emergency platform. Information construction, 9, 14-17.

[10] Weicheng Fan,Yi liu\&Wengguo Weng.(2009), the framework of the "triangle theory " and " $4+1$ "methodology for the public safety technology.Technology Review,6,1.

[11] Yueqiang Fu.(2008) response reliability theory and its application in the fire emergency.nanchang university.

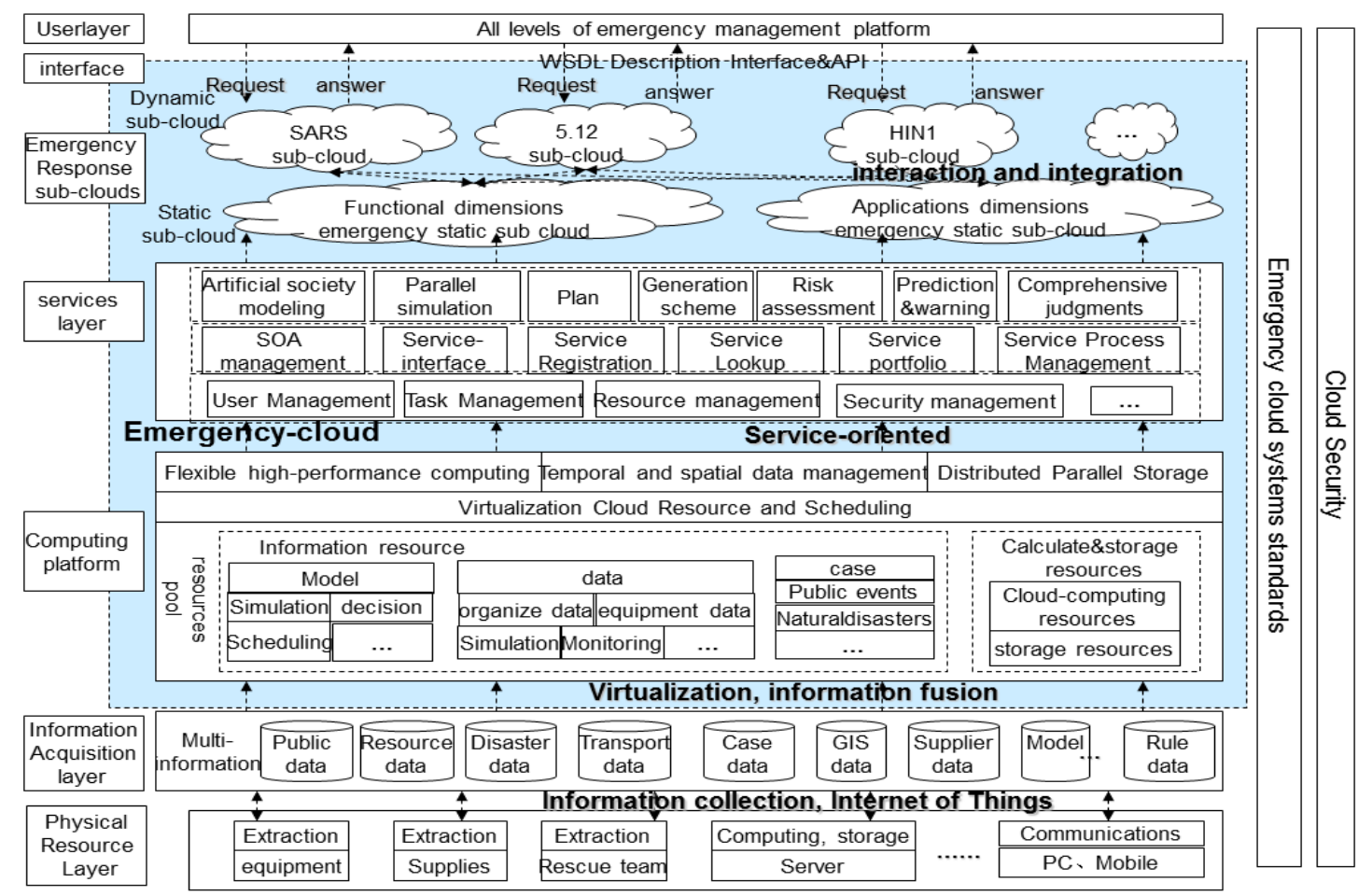

Firture2.The architecture of Emergency-Cloud 